\title{
Integrating Health Belief Model, Technological Self-efficacy, and the PEN-3 cultural model: Conceptualizing the impact of culture and technology on health for advancing Sustainable Smart Health
}

\author{
Xue $\mathrm{Wu}^{1}$, Man Zhao ${ }^{1}$, Han-Teng Liao ${ }^{1 *}$ \\ ${ }^{1}$ New Media Research Centre, Sun Yat-sen University Nanfang College, Guangzhou, Guangdong, 510970, China
}

\begin{abstract}
As people record, visualize, analyze, share, reflect on, etc. their everyday life using digital and network technologies, how can researchers and designers empower them to engage both the technologies and health about themselves? Though the Health Belief Model (HBM) has been used to explain and predict healthrelated behaviors, and the Technological Self-efficacy (TSE), and the PEN-3 cultural model has been used as constructs of technological and cultural self-efficacy, it remains a challenging task to tease out the impact of cultural and technological factors for people to improve their health conditions and well-being by taking direct and indirect actions. With the aim to develop a conceptual framework to overcome such a challenge, this study examined and selected a few constructs from the TSE and PEN-3 cultural models, respectively, and then use them to enrich the HBM so that the impact of cultural and technological factors can be better integrated and examined. The integrated model can be used as an analysis tool for both researchers and designers to identify first the relevant cultural and technological factors (using selected constructs), and then formulate and then test hypotheses regarding how these factors shape their health and technology actions (using the causal modeling of the enriched HBM). The integrated model proposed and illustrated in this study shows the ways in which both cultural and technological factors can be conceptualized to explain and predict health-related behaviors via perceived beliefs (often related to technology and health). For example, self-tracking visualization involves both cultural and technological factors that may facilitate or impede health-related behaviors.
\end{abstract}

\section{INTRODUCTION}

As people record, visualize, analyze, share, reflect, etc. on their everyday life using digital and network technologies, how can researchers and designers empower them to engage both the technologies and health about themselves? As a mixture of making, thinking, contextualizing and envisioning, design should create tangible propositions for the mutual benefit of both user and manufacturer, both human and technology[1]. Some design and user research efforts have been made to put the perspective of the user and digital product designers in the context of individual and social well-being outcomes[2,3]. Others searched for basic principles and theories to design endeavor from philosophy and psychology, proposing that designers and HCI people should value human body as bodily experience improves reflective capacities[4], therefor, adopt the aesthetics of technologies emerge out of a dynamic interaction between a user and an interactive system[5]. Such research aims to figure out the context of technology design for people and the way it enriched our daily lives with self-presentation, culture preference and social being.

However, self-tracking and related technologies have been highly rejected and abandoned by users[6,7]. Research had been done to investigate the particular role of privacy concerns in continuous usage of fitness trackers[8]; the factors that lead to continuous use of selftracking devices had also been concluded through empirically analyzation[9]. Such past studies adopted HBM and TSE independently to explain internet use for health-related behaviors. However, they lack the ability to explain comprehensive relationships among health belief, technology belief, and culture belief in explaining users' behaviors of health technology.

With the aim to develop a conceptual framework to overcome such a challenge, this study examined and selected a few constructs from the TSE and PEN-3 cultural models, respectively, and then use them to enrich the HBM so that the impact of cultural and technological factors can be better integrated and examined. This paper will first provide a background and review of related work. Then it will combine TSE and PEN-3 culture model with traditional $\mathrm{HBM}$, in order to investigate the causality 
among health belief, technology belief, and culture belief to action of adopt/refuse of health technology.

\section{BACKGROUND AND RELATED WORK}

\subsection{Health Belief Model}

Self-efficacy has been deeply discussed as it predicted a range of health behaviors and is often included in other health behavior models, including the Health Belief Model (HBM), where it has improved the predictive efficacy through the HBM and personal health behavior, the role of behavioral science theory in development and implementation of public health interventions, and historical origins of the HBM[8,10-12]. As one of the most widely applied theories of health behavior[13], the self-efficacy HBM has been successfully adapted to fit diverse cultural and topical contexts[14].

One successful approach to improving health behaviors has been the development of interventions based on a sociologic approach that takes into account people's cultural beliefs, attitudes, and behaviors[15], as well as the involvement of diverse social relationships, for example, lay health educators in the context of an educational program[16,17]. Further research should be done to exam the exact connotation and effect of such social elements of users' behaviors using technologies through self-efficacy HBM. The remaining of the paper seeks to propose a Theoretical Technological HBM for Self-tracking in designing self-tracking systems. First, it generalized a theoretical model to incorporate technological self-efficacy into HBM. Then based on the theoretical model, it generalizes what elements are influencing people's behavior when using self-tracking technology, and explores the elements that may be influencing such behavior.

\subsection{Technological self-efficacy}

Social Cognitive Theory (SCT) is common model used to explain users' social behavior, which states that behavior is a function of individuals' expectations of the consequences of the action, their abilities to execute the action, and their beliefs that the action will achieve a desired outcome[18]. Among which self-efficacy has been deeply discussed as it predicted a range of health behaviors $[19,20]$.

As possible explanations for the acceptance or rejection of technological innovations have become a crucial topic in research, technological self-efficacy (TSE) becomes a crucial factor affects acceptance motives of different types of technology. TSE is one's perceived ability to use a product successfully[21], one's expectations of personal mastery and success[22], and one's confidence in ability to perform specific tasks[23], which refers to a key variable for explaining users' acceptance of technology infrastructure[24]. Educational researchers have employed TSE in a wide range of academic and technology-related settings and have found positive and strong influence of efficacy beliefs on achievement and persistence regarding specific, criterial tasks[25,26]. Such research had help explain individual approval, discomfort, or residence towards technical in certain scenarios.

However, TSE is rarely used to explain the user behavior of a category of technology products, but more to investigate the relationship between general concept of technology, other elements and user behavior in certain contexts.

\subsection{PEN-3 Culture Model}

Over the past decade, available evidence focusing on the impact of culture on health has increased dramatically. This indicates not only a widespread and growing interest in the influence of culture but also the realization of its importance in eliminating health disparities, addressing health literacy, and designing and implementing effective public health interventions[27]. One significant model that has been at the forefront of understanding the influence of culture on health is the PEN-3 cultural model[28]. The PEN-3 cultural model consists of three primary domains: (1) Cultural Identity, (2) Relationships and Expectations, and (3) Cultural Empowerment. Each domain includes three factors that form the acronym PEN; Person, Extended Family, Neighborhood (Cultural Identity domain); Perceptions, Enablers, and Nurturers (relationship and expectation domain); Positive, Existential and Negative (Cultural Empowerment domain)[27](See Fig.1).The PEN-3 culture model as an organizing frame to centralize culture when defining health problems and framing their solutions has been adopted by researchers mainly from public health interventions[14,29].

\section{THE HBM COMBINES WITH TSE AND PEN-3 CULTURE MODEL}

\subsection{Combines HBM with TSE}

The HBM suggests that a person's belief in a personal threat of an illness or disease together with a person's belief in the effectiveness of the recommended health behavior or action will predict the likelihood the person will adopt the behavior [30]. As shown in Figure.2, besides basic demographic variables and psychological characteristics, elements like perceived susceptibility, perceived severity, health motivation, perceived benefits, perceived barriers, and cues to action are included in the model, which are commonly used to health management proposal by researchers of diverse fields such as information management, social commerce, culture, etc.[10,14,31] 


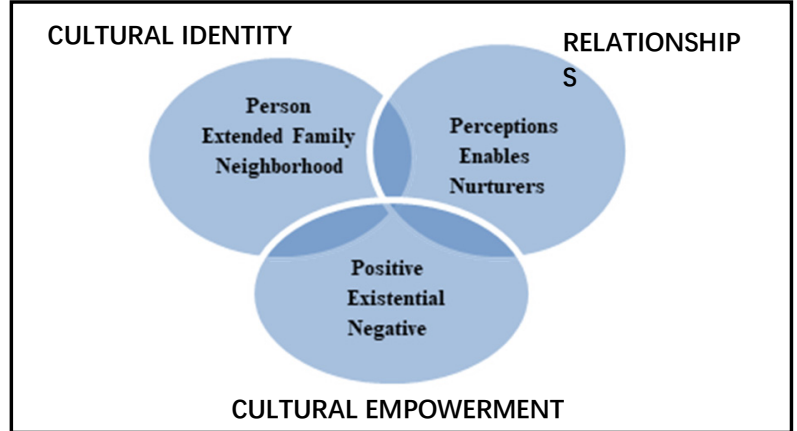

Fig1. The original PEN-3 Cultural Model. (Bowered from Airhihenbuwa[28])

Technological Self-efficacy (TSE) was first discussed in Pam Scholder Ellen's research on the factors cause individual resistance to technological innovations. Ellen proposed variables as criterion resistance, manipulation checks satisfaction, self-efficacy, outcome expectancy, performance attribution, covariates intrinsic satisfaction, and innovativeness are proposed, with results could indicate that a person's perceived ability to use a product successfully affects their evaluative and behavioral response to the product[21].

As TSE suggests one's confidence in ability to perform specific tasks, or one's perceived ability to use a product successfully, it is also a discussion of one's belief and ones' (technological) behavior or action, which makes it possible to combine TSE into HBM, especially when discussing a person's belief in a personal threat of technology together with a person's belief in the effectiveness of the recommended technological behavior or action will predict the likelihood the person will adopt the technology.

As shown in Figure. 3, variables as perceived selfefficacy, perceived technological benefits, and perceived

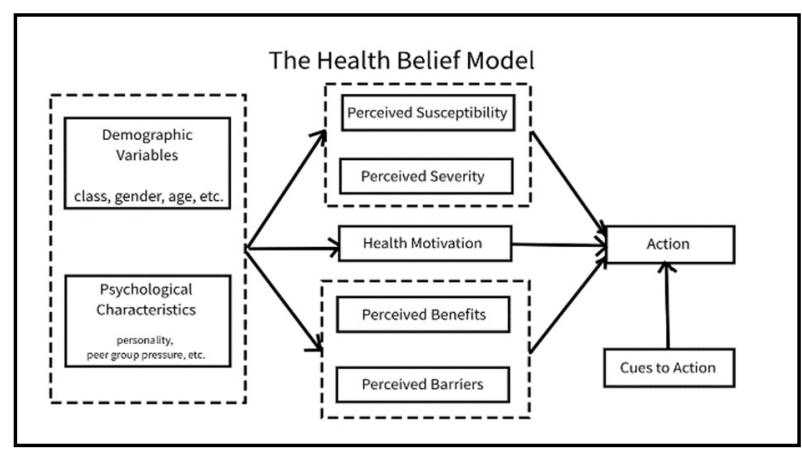

Fig2. The common Health Belief Model (HBM). (Bowered from Rosenstock [8] )

technological barriers are added to the original HBM, as these variables are very likely to influence individual's behaviors to technology. These three variables summarize and includes those variables of Pam Scholder Ellen's research. "Perceived self-efficacy" includes the original "self-efficacy", which indicates people's belief in selfefficacy; "Perceived Technological Benefits" indicates a person's perception of the effectiveness of various actions available to successfully use technology, which summarized the core concepts of some of the original variables from Ellen ("manipulation checks satisfaction", "covariates intrinsic satisfaction", "innovativeness", "outcome expectancy", and "performance attribution"). "Perceived Technological Barriers" refers to a person's feelings on the obstacles to performing a technological action. There is wide variation in a person's feelings of barriers, or impediments, which lead to a cost/benefit analysis. It also summarized the core concepts of some of the original variables ("criterion resistance", "outcome expectancy", and "performance attribution").

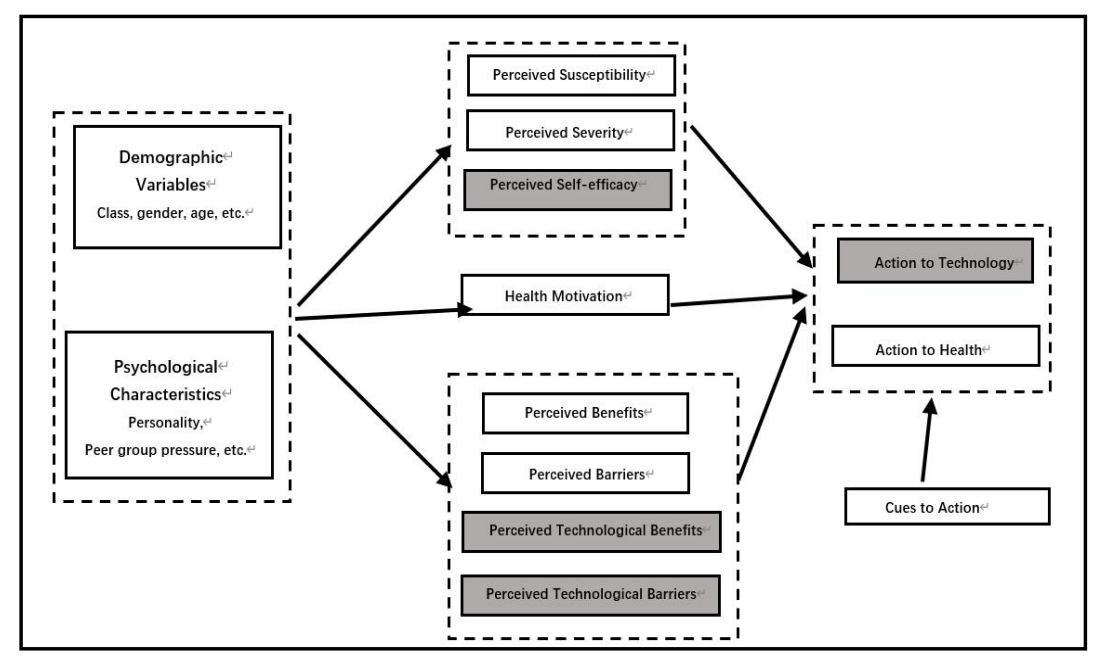

Fig3. The Health Belief Model combines with Technological Self-efficacy

There would be two types of action as consequence in this model, "Action to Technology" and "Action to Health". The traditional HBM suggests the causality between a person's belief in in an illness or disease and health behavior or action. By combining with TSE, variables could result in people's action either to health or technology, or both. 


\subsection{Combines PEN-3 Culture Model with HBM}

As human behaviors to health and technology are a comprehensive consequence of mixture elements includes culture, some research had investigated norms of a certain cultures and human actions to health. Scarinci combines the PEN-3 cultural model and HBM as theoretical guidance for the intervention development and implementation of prevention of cervical cancer among Latina immigrants, which expanded the original HBM to a wider cultural context[14].

According to Iwelunmor, the PEN-3 cultural model highlights the impact of behavior on health (positive, existential, or negative), the key influences of the behavior (perceptions, enablers, or nurturers), and the focus of the health behavior intervention (person, extended family, or neighborhood)[27], as shown in Figure 1.In the HBM combines with TSE above in Figure 2, we combined TSE to HBM to investigate whether technological belief affects healthy behavior or healthy technological behavior. In this step, we would add cultural considerations, because both culture belief and technological belief may affect healthy behavior. In order to know how cultural belief, technological belief and health belief affect healthy / unhealthy technological behavior in whole or in part, the relationship between culture and technology need to be studied. The traditional PEN-3 culture model is not a causality diagram but "an organizing frame to centralize culture when defining health problems and framing their solutions"[27]. In this paper, we try to integrate culture elements into HBM. The expanded HBM combines with TSE and PEN-3 is shown in Figure 4.

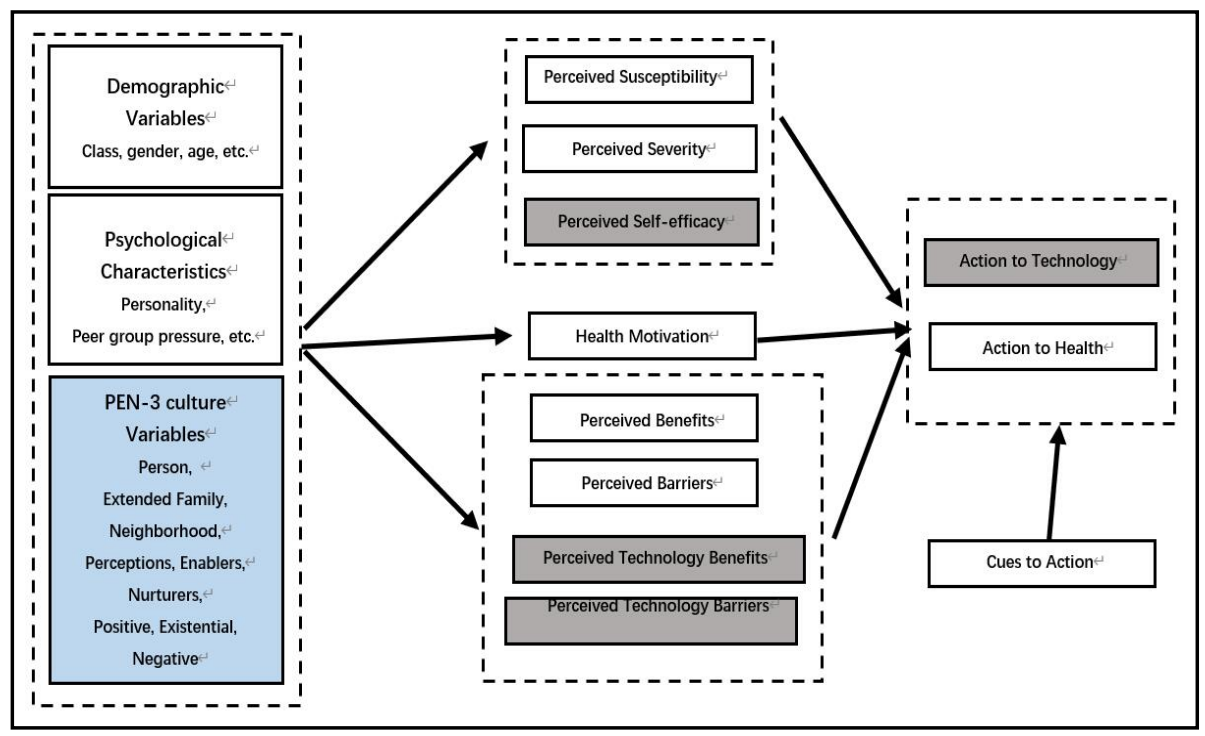

Fig4. The HBM combines with TSE and PEN-3

\section{CONCLUSION}

The main contribution of this paper is combining TSE and PEN-3 culture model with traditional HBM, in order to investigate the causality among health belief, technology belief, and culture belief to action of adopt/refuse of health technology. This paper enriches the HBM so that the impact of cultural and technological factors can be better integrated and examined, as it tries to complete a challenging task to tease out the impact of cultural and technological factors for people to improve their health conditions and well-being by taking direct and indirect actions. The integrated model proposed and illustrated in this study shows the ways in which both cultural and technological factors can be conceptualized to explain and predict health-related behaviors via perceived beliefs (often related to technology and health).

We will continue our research with a quantitativeempirical evaluation base on the model. With our research, we aim at contributing to both a better theoretical understanding in the field of technology for well-being in a social context and giving practical implications for producers of daily-used technologies such as self-tracking, moreover, to propose a conception of Sustainable Smart Health., which align design to individual desire for selfimprovement through self-presentation, and to the behavior changes that make healthier and caring individuals and a more sustainable built or natural environment. Although future empirical work must be conducted to see whether and how well the integrated model works, the study nevertheless has facilitated more systematic and holistic understanding of the impact of culture and technologies for health sustaining activities, the key question to advance what we call Sustainable Smart Health.

\section{ACKNOWLEDGMENTS}

The research is funded by the Application-oriented Curriculum Development Project of "Information Visualization Design" (NFU 02-40250), under the Guangdong Province Department of Education 2018 "Innovation-Strengthening Higher Education Program" Grants, and partly by a project of HCI for Education Development (2018WQNCX283), under the 2018 Major 
Projects of Guangdong Province Department of Education "Young Innovative Talents" Grants.

\section{REFERENCE}

1. LOWGREN, J., CARROLL, J.M., HASSENZAHL, M., ERICKSON, T. Industrial Design by Kees Overbees and Caroline Hummels - The Encyclopedia of Human-Computer Interaction, 2nd Ed. (Interaction Design Foundation)

2. Casais, M., Mugge, R., Desmet, P. (2018) Objects with symbolic meaning: 16 directions to inspire design for well-being. J Des. Res. 16: 247.

3. Banos, O., Bilal Amin, M., Ali Khan, W., Afzal, M., Hussain, M., Kang, B.H., Lee, S. (2016) The Mining Minds digital health and wellness framework. Biomed. Eng. OnLine 15: 76.

4. Maurice, M.-P. (1958) Phenomenology of Perception. (London, England,Routledge)

5. Locher, P., Overbeeke, K., Wensveen, S. (2010) Aesthetic Interaction: A Framework. Des. Issues 26: 70-9.

6. Hocking, C. (1999) Function or feelings: factors in abandonment of assistive devices. Technol. Disabil. 11: 3-11.

7. Ledger, D., McCaffrey, D. (2014) Inside wearables: How the science of human behavior change offers the secret to long-term engagement. Endeav. Partn. 200: 1.

8. Rosenstock, I.M., Strecher, V.J., Becker, M.H. (1988) Social Learning Theory and the Health Belief Model. Health Educ. Q. 15: 175-83.

9. Buchwald, A., Letner, A., Urbach, N. Towards Explaining the Use of Self-Tracking Devices: Conceptual Development of a Continuance and Discontinuance Model. 12.

10. Norman, P., Brain, K. (2005) An application of an extended health belief model to the prediction of breast self-examination among women with a family history of breast cancer. Br. J. Health Psychol. 10: 116.

11. Wdowik, M.J., Kendall, P.A., Harris, M.A., Auld, G. (2001) Expanded Health Belief Model Predicts Diabetes Self-Management in College Students. J. Nutr. Educ. 33: 17-23.

12. Webb, T.L., Sheeran, P. (2006) Does changing behavioral intentions engender behavior change? A meta-analysis of the experimental evidence. Psychol. Bull. 132: 249-68.

13. Glanz, K., Bishop, D.B. (2010) The Role of Behavioral Science Theory in Development and Implementation of Public Health Interventions. Annu. Rev. Public Health 31: 399-418.

14. Scarinci, I.C., Bandura, L., Hidalgo, B., Cherrington, A. (2012) Development of a Theory-Based (PEN-3 and Health Belief Model), Culturally Relevant Intervention on Cervical Cancer Prevention Among Latina Immigrants Using Intervention Mapping.
Health Promot. Pract. 13: 29-40.

15. Valdez, A., Banerjee, K., Ackerson, L., Fernandez, M. (2002) A multimedia breast cancer education intervention for low-income Latinas. J. Community Health 27: 33-51.

16. Fernández, M.E., Diamond, P.M., Rakowski, W., Gonzales, A., Tortolero-Luna, G., Williams, J., Morales-Campos, D.Y. (2009) Development and Validation of a Cervical Cancer Screening SelfEfficacy Scale for Low-Income Mexican American Women. Cancer Epidemiol. Biomark. Prev. Publ. Am. Assoc. Cancer Res. Cosponsored Am. Soc. Prev. Oncol. 18: 866-75.

17. Navarro, A.M., Senn, K.L., McNicholas, L.J., Kaplan, R.M., Roppé, B., Campo, M.C. (1998) Por La Vida model intervention enhances use of cancer screening tests among Latinas. Am. J. Prev. Med. 15: 32-41.

18. Bandura, A. (1978) Self-efficacy: Toward a unifying theory of behavioral change. Adv. Behav. Res. Ther. 1: 139-61.

19. Schüz, B., Sniehotta, F.F., Wiedemann, A., Seemann, R. (2006) Adherence to a daily flossing regimen in university students: effects of planning when, where, how and what to do in the face of barriers. J. Clin. Periodontol. 33: 612-9.

20. Gochman, D.S. (1997) Handbook of Health Behavior Research II - Provider Determinants. (New York: Plenum)

21. Ellen, P.S., Bearden, W.O., Sharma, S. (1991) Resistance to technological innovations: An examination of the role of self-efficacy and performance satisfaction. J. Acad. Mark. Sci. 19: 297-307.

22. Sherer, M., Maddux, J.E., Mercandante, B., PrenticeDunn, S., Jacobs, B., Rogers, R.W. (1982) The SelfEfficacy Scale: Construction and Validation. Psychol. Rep. 51: 663-71.

23. Bandura, A. (1977) Self-efficacy: Toward a unifying theory of behavioral change. Psychol. Rev. 84: 191215.

24. Zaunbrecher, B.S., Kowalewski, S., Ziefle, M. (2014) The Willingness to Adopt Technologies: A CrossSectional Study on the Influence of Technical Selfefficacy on Acceptance. Human-Computer Interaction. Applications and Services vol 8512, ed M Kurosu (Cham: Springer International Publishing) pp 764-75

25. Farah, A.C. (2012) Factors Influencing Teachers' Technology Self-Efficacy: A Case Study. (ProQuest LLC)

26. Bose, I.C., Olanrewaju, A.K., Bamidele, O. TECHNOLOGY USE AND SELF-EFFICACY AS PREDICTORS OF ACADEMIC STANDING AMONG POLYTECHNIC STUDENTS IN OGUN STATE.

27. Iwelunmor, J., Newsome, V., Airhihenbuwa, C.O. (2014) Framing the impact of culture on health: a systematic review of the PEN-3 cultural model and its 
application in public health research and interventions. Ethn. Health 19: 20-46.

28. Airhihenbuwa, C.O. (1989) Perspectives on AIDS in Africa: strategies for prevention and control. AIDS Educ. Prev. Off. Publ. Int. Soc. AIDS Educ. 1: 57-69.

29. Airhihenbuwa, C., Okoror, T., Shefer, T., Brown, D., Iwelunmor, J., Smith, E., Adam, M., Simbayi, L., Zungu, N., Dlakulu, R., Shisana, O. (2009) Stigma, Culture, and HIV and AIDS in the Western Cape, South Africa: An Application of the PEN-3 Cultural Model for Community-Based Research. J. Black Psychol. 35: 407-32.

30. Jones, C.L., Jensen, J.D., Scherr, C.L., Brown, N.R., Christy, K., Weaver, J. (2015) The Health Belief Model as an Explanatory Framework in Communication Research: Exploring Parallel, Serial, and Moderated Mediation. Health Commun. 30: 56676.

31. CHEN, Y., CHEN, X., GU, T. (2019) Research on the Influence of Expected Confirmations on the Willingness of Continuous Use of Online Knowledge Q\&A Community under the Framework of ECM-ISC ECM-ISC. Agric. Libr. Inf. 